\title{
REFORMA EDUCATIVA EN SECUNDARIA Y APRENDIZAJE POR COMPETENCIAS
}

\section{RESUMEN}

\author{
Dra. Emma Leticia Canales Rodríguez * \\ "No basta con saber, también hay que aplicar. \\ No basta con querer, también hay que actuar".
}

Goethe

Este trabajo presenta algunos elementos de análisis del Plan de Estudios para la Educación Secundaria que se establece en el Acuerdo 384 en torno a las cinco competencias que se proponen para lograr el perfil de egreso que se espera alcanzar desde todas las asignaturas con motivo de la Reforma Educativa 2006.

Resalta la necesidad de entender esta propuesta desde la propia concepción del aprendizaje como proceso y de la evaluación de los mismos. Destaca la importancia de advertir las diferentes formas de enseñar y aprender que han prevalecido en diferentes momentos de nuestra historia para entender la necesidad de formar alumnos reflexivos, críticos y autónomos. Ofrece desde la visión de varios autores las implicaciones didácticas que implica el desarrollo de programas bajo este paradigma.

\section{ABSTRACT}

This work presents some elements of analysis of the Curriculum for Secondary Education that is established in the Agreement 384 around the five competitions set out to obtain the graduation profile that is hoped to be reached from all the subjects on the occasion of the Educational Reform 2006. The necessity to understand this proposal stands out from the own conception of learning as a process and the evaluation of the same. It emphasizes the importance of noticing the different ways to teach and to learn that have prevailed at different moments in our history to understand the necessity to train reflective, independent and critic students. It offers, from the vision of several authors, the didactic implications that the development of programs under this paradigm imply.

*Doctora en Educación de la Universidad Autónoma de Tlaxcala, Maestra en Psicología Social por la UNAM y Licenciada en Psicología por la UNAM. Actualmente es Profesor Investigador de tiempo completo en el Área Académica de Ciencias de la Educación de la Universidad Autónoma del Estado de Hidalgo (Instituto de Ciencias Sociales y Humanidades).Coautor de numerosas publicaciones entre las que destacan: Activación del Pensamiento en Educación Básica, libro de recursos y edición anotada para el profesor (9 libros de primaria y secundaria) Serie 2000, Santillana, 2001. Coordinadora de los libros: La Tutoría un Asunto para la Reflexión y Cultura Escolar, intenciones y transición de los alumnos de licenciatura de la Universidad Autónoma de Tamaulipas (2002 Universidad Autónoma de Tamaulipas y UAEH), La tutoría académica, un espacio para la reflexión (2002, UAEH- UATx). La formación de tutores académicos en educación superior (2004), CESU-UNAM; Tutoría Integral y educación de calidad en la educación superior (2005), Universidad de Valencia, Orientación y tutoría para alumnos de secundaria, 2007. 


\section{INTRODUCCIÓN}

La educación contribuye a la formación integral de los sujetos en múltiples facetas: mejorar su calidad de vida, facilitar la adquisición de conocimientos, habilidades y actitudes que poseen un valor intrínseco que les permite acrecentar su libertad, fortalecer sus valores y tener un panorama más amplio para tomar diferentes decisiones a lo largo de su vida.

Con la Reforma Educativa de Secundaria la responsabilidad adquirida por la mayoría de los docentes de este tramo educativo adquiere gran importancia. De ellos depende tomar con seriedad los contenidos y estrategias que contempla y aunque ha provocado una gran polémica desde su inicio por la complejidad que representa, es una realidad en el último tramo de la educación básica.

Una de las características que tiene el programa es el énfasis en el desarrollo de competencias y la definición de aprendizajes esperados.

El eje de este trabajo se enfoca en las competencias que propone el plan de estudios para contribuir al logro del perfil de egreso de los alumnos al concluir su secundaria. Para abordarlo, se presentan los siguientes apartados: inicia con una contextualización del proceso de enseñanza en diferentes períodos de la historia, su propósito es entender la razón por la que ahora, en el siglo XXI se considera la formación en competencias en los rediseños curriculares de diferentes niveles educativos como el adecuado para formar a los y las jóvenes.

En el segundo apartado, que se desprende del anterior, se identifican los factores que intervienen en el aprendizaje, mismos que dan pie para el último apartado, donde se plantea la formación en competencias. A lo largo del trabajo se van entretejiendo las propuestas que aparecen en el Plan de Estudios para la Secundaria aprobado en marzo del 2006 con motivo de la Reforma Educativa de Secundaria. Con la información que se ofrece se cierra con algunas reflexiones finales en torno a la necesidad de formar a los docentes que están educando en competencias.

I.- Contexto del proceso de enseñanza aprendizaje

Toda intervención docente es una respuesta al problema básico de cómo abordar adecuadamente la enseñanza y al parecer es una cuestión que no parece estar ni mucho menos resuelta en la actualidad y cuya aclaración resulta en estos momentos insoslayable para superar los obstáculos que dificultan la mejora de la enseñanza en los diferentes niveles educativos.

Enseñar resulta una problemática compleja que se ha abordado de diferentes formas a lo largo de nuestra historia y ha estado presente en la práctica pedagógica de la que se desprenden roles específicos que asumen los que enseñan y los que "aprenden". 
En la actualidad, se considera que el papel del profesor en el acto didáctico se centra en proveer de recursos y entornos diversificados de aprendizaje a sus estudiantes. Para ello les motiva para que le den sentido a lo que aprenden, les ofrece orientación a lo largo de su proceso de aprendizaje y los asesora para apoyarlos a realizar sus trabajos y tareas.

Sin embargo, a lo largo del tiempo la enseñanza se ha realizado desde otras concepciones y de ahí que los roles de los profesores y alumnos han sido distintos.

Ha habido una transformación de la educación desde la "pedagogía de la reproducción" a la "pedagogía de la imaginación" que se sustenta más en la indagación y la búsqueda que en la respuesta (Beltrán Llera, 2003); de estar centrada en la enseñanza y el profesor a centrarse en el aprendizaje y el alumno; de atender sobre todo a los productos a considerar la importancia de los procesos. A grandes rasgos, las principales visiones sobre la enseñanza, que han ido evolucionando de manera paralela sobre las concepciones del aprendizaje pueden concretarse de la siguiente manera:

a) Modelo didáctico-expositivo, se refiere a la clase magistral expositiva.

Se desarrolla en una época en la que muy pocos accedían a la cultura, el profesor (en la universidad o como tutor de la familia) era prácticamente el único proveedor de información que tenían los estudiantes. La clase magistral era la técnica de enseñanza más común. Estaba centrada en el profesor y el aprendizaje buscaba la memorización de los saberes que transmitía el maestro de manera sistemática y estructurada.

b) Modelo didáctico-instructivo, corresponde a la clase magistral y el libro de texto. Con el tiempo, la difusión de los libros se amplió, se crearon bibliotecas, la cultura se hizo extensiva a diferentes grupos sociales y los libros estuvieron presentes. El profesor se consideraba el máximo depositario de la información que recibían los alumnos a pesar de la existencia de diferentes pensadores sobre temas pedagógicos que defendían ideas distintas. El profesor era un instructor y la enseñanza estaba centrada en los contenidos que el alumno debía memorizar y aplicar para contestar preguntas y realizar ejercicios que le ayudarían a asimilar los contenidos.

c) Modelo didáctico-alumno activo, se comienza a aplicar en la escuela activa y se empiezan a aceptar en las instituciones educativas las enseñanzas de la "escuela activa" (Dewey, Freinet, Montessori). Se considera que el alumno no debe conformarse con mantener una actitud pasiva y receptiva. Se empieza a promover que los estudiantes desarrollen proyectos que les permitan descubrir el conocimiento, aplicarlo en situaciones prácticas y desarrollar todas sus actividades. La enseñanza se centra en la actividad del alumno que a menudo debe ampliar y reestructurar sus conocimientos para poder hacer frente a las problemáticas que se le presentan.

A pesar del desarrollo y las propuestas de modelos pedagógicos interactivos, coexistió el modelo memorístico anterior basado en la clase magistral del profesor y el estudio del libro de texto, completando todo ello con la realización de ejercicios generalmente rutinarios y repetitivos. 
d) Modelo didáctico-colaborativo, se enfoca en la enseñanza abierta y colaborativa. A finales del siglo XX, los grandes avances tecnológicos y el triunfo de la globalización económica y cultural, configura una nueva sociedad, la sociedad "de la información". En este marco, con el acceso cada vez más generalizado de los ciudadanos a los "medios masivos" e Internet, proveedores de todo tipo de información, y pudiendo disponer de unos versátiles instrumentos para realizar todo tipo de procesos con la información (las computadoras), se va dando paso a un nuevo currículo básico para los ciudadanos y un nuevo paradigma de la enseñanza:

"la enseñanza abierta". En este nuevo paradigma, heredero de los principios fundamentales de la escuela activa, cambia el rol del profesor, quien disminuye su papel como transmisor de información. Presenta los temas de manera contextualizada, enfatiza en los aspectos más importantes o de difícil comprensión, destaca sus aplicaciones, motiva a los alumnos a que sean más autónomos en su estudio. Por otro lado los estudiantes pueden acceder con mayor facilidad a cualquier clase de información, de tal forma que el docente pasa a ser un orientador de sus aprendizajes, proveedor y asesor de los recursos educativos más adecuados para cada situación, organizador de entornos de aprendizaje, tutor, consultor (Tebar, 2003).

Cuadro 1. Roles y Modelos, cien años de historia (Beltrán Llera, 2003

\begin{tabular}{|c|c|c|c|}
\hline Año & El que enseña & El que aprende & Modelo \\
\hline 1900 & $\begin{array}{l}\text { Profesor } \\
\text { Dice, habla }\end{array}$ & $\begin{array}{l}\text { Alumno } \\
\text { escucha, memoriza }\end{array}$ & \multirow{2}{*}{$\begin{array}{l}\text { Modelo didáctico expositivo } \\
\text { centrado en el profesor }\end{array}$} \\
\hline 1925 & $\begin{array}{l}\text { Maestro } \\
\text { explca }\end{array}$ & $\begin{array}{l}\text { Estudiante } \\
\text { entiende, memoriza }\end{array}$ & \\
\hline 1950 & $\begin{array}{l}\text { Docente } \\
\text { instruye, demuestra }\end{array}$ & $\begin{array}{l}\text { Dicente } \\
\text { experimenta y aplica }\end{array}$ & $\begin{array}{l}\text { Modelo didáctico instructivo } \\
\text { centrado en los contenidos }\end{array}$ \\
\hline 1975 & $\begin{array}{l}\text { Educador } \\
\text { construye }\end{array}$ & $\begin{array}{l}\text { Educando } \\
\text { aprende }\end{array}$ & $\begin{array}{l}\text { Modelo didáctico centrado } \\
\text { en la actividad del alumno }\end{array}$ \\
\hline 2000 & $\begin{array}{l}\text { Mediador } \\
\text { transforma }\end{array}$ & $\begin{array}{l}\text { Lider transformador } \\
\text { compite }\end{array}$ & $\begin{array}{l}\text { Modelo didáctico colaborativo } \\
\text { centrado en ambos }\end{array}$ \\
\hline
\end{tabular}


Es así que a principios del siglo XXI, "Ya no basta con que cada individuo acumule al comienzo de su vida una reserva de conocimientos a la que podrá recurrir después sin límites. Sobre todo debe estar en condiciones de aprovechar y utilizar durante toda la vida cada oportunidad que se le presente de actualizar, profundizar y enriquecer ese primer saber y de adaptarse a un mundo en permanente cambio" (Delors, 1996; 95). Los objetivos y resultados del proceso de formación se establecen como: Aprender a conocer, que implica adquirir los conocimientos de la comprensión; Aprender a hacer para poder influir sobre el propio entorno; Aprender a vivir juntos para participar y cooperar con los demás y Aprender a ser que implica un proceso que encierra a los otros tres. Éstos pasan a ser una parte importante de los cambios en los sistemas de enseñanza aprendizaje (Delors, 1996).

Se aconseja a través de diferentes publicaciones entre 1995 y 2002, unir esfuerzos a partir de enseñar a aprender; Libro Blanco sobre Educación y Formación: Enseñar y Aprender. Hacia la Sociedad Cognitiva (1995); el Libro Verde: Vivir y Trabajar en la sociedad de la información: prioridades para las personas (1996); Plan de acción para el empleo en el reino de España (1999); Memorando sobre aprendizaje permanente (2000); Ley de cualificaciones y de Formación Profesional (2002). Las recomendaciones que aquí se reúnen están vinculadas con los cambios en la lógica de producción y en las organizaciones que se basan en la cooperación y el interés común.

Con el modelo didáctico colaborativo, se empiezan a diseñar las propuestas curriculares basadas en competencias que enfatizan la educación profesional, y que proporcionan los fundamentos para incorporarse a la vida laboral.

La preparación que esta concepción curricular ofrece forma parte de los planteamientos considerados en el Plan de Estudios que propuso la Reforma de la Secundaria en marzo del 2006. Ésta empezó a operar con alumnos de primero a partir del ciclo escolar 2006-2007 y en este momento cursan este nuevo plan también los alumnos de $2^{\circ}$ de secundaria.

En el último tramo de la educación básica, se pretende introducir al alumnado en el mundo de la ciencia, la tecnología y la técnica donde confluyen los sistemas laborales a partir de la fundamentación de una estructura cognitiva en el que se asienta la adquisición posterior de conocimientos, habilidades, actitudes más específicas, permitiendo a la vez que esa estructura sea lo suficientemente amplia como para asimilar los cambios posteriores que deberá asumir la persona a lo largo de su vida laboral.

En este tenor, el énfasis en el desarrollo de competencias de la propuesta curricular de la Reforma Educativa de Secundaria, plantea que, para alcanzar el desarrollo esperado en los perfiles de egreso, propiciará a lo largo de sus estudios que los alumnos movilicen sus saberes dentro y fuera de la escuela (Acuerdo 384). Este tema lo retomaremos más adelante, al debatir sobre las dificultades que enfrenta el sistema educativo para "movilizar los saberes de sus alumnos". 
II. Factores que influyen en el aprendizaje.

Un aspecto importante para desarrollar una competencia se relaciona con los factores que influyen en el aprendizaje, a saber las actitudes, que están relacionadas con lo que se quiere aprender, las aptitudes que reflejan lo que se puede aprender y los contenidos que se traducen en lo que se aprende. Las actitudes son una predisposición afectiva y motivacional requerida para el desarrollo de una determinada acción, poseen también un componente cognitivo conductual. En la actitud lo fundamental es generar expectativa, porque así el estudiante se interesa y motiva en su proceso de aprendizaje y así es posible que incremente su atención y comprensión.

Las aptitudes se refieren a la capacidad para pensar y para actuar y se desarrollan en los tres ámbitos de los contenidos de aprendizaje: la primera se conoce como aptitud intelectiva, se refiere a las habilidades mentales que determinan el potencial de aprendizaje, también definidas como las capacidades para pensar y saber. Dependen de la estructura mental, las funciones cognitivas, los procesos de pensamiento y las inteligencias múltiples. Las aptitudes para actuar se denominan procedimentales y se definen como las capacidades para actuar y hacer. Están relacionadas con los métodos, técnicas, procesos y estrategias empleadas en el desempeño y se traducen en hábitos, habilidades y destrezas.

Están relacionadas con la calidad del desempeño.

Los contendidos se refieren a toda la estructura conceptual susceptible de ser aprendida. Su organización es vital para el proceso de aprendizaje.

La comprensión de los conceptos determina el aprendizaje, mas no el significativo. En la medida en que exista más coherencia entre ellos, los estudiantes encontrarán relaciones entre los mismos lo que a su vez aumentará su nivel de comprensión. Están implícitos en el currículo y se desarrollan por áreas, unidades de aprendizaje o asignaturas que cuentan con núcleos temáticos organizados a través de diferentes conceptos que incluyen los temas.

Los contenidos que se enseñan en todos los niveles educativos se agrupan en tres áreas básicas:

Conocimiento declarativo (saber que), conocimiento procedimental (saber hacer) y conocimiento actitudinal (saber ser).

El aprendizaje de contenidos declarativos, se refiere al aprendizaje de hechos, conceptos y principios. Significa que el que aprende es capaz de reconocer, identificar, describir y comparar objetos, sucesos e ideas.

El aprendizaje de contenidos procedimentales, significa aprender un método que el sujeto es capaz de utilizarlo en diversas situaciones y de diferentes maneras, con el fin de resolver problemas planteados para alcanzar las metas fijadas.

El aprendizaje de contenidos actitudinal-valorales, significa que el que aprende es capaz de regular el propio comportamiento de acuerdo con el principio normativo que dicho valor estipula, está relacionado con los valores, normas y actitudes. (Díaz Barriga, 2001).

De lo anterior se desprende que quien sabe actuar, y lo hace bien es porque además de que tiene un dominio de contenidos declarativos 
(saber que), comprende cómo funciona su pensamiento (sabe hacer) y cómo se interrelacionan los conceptos en ese proceso de aprendizaje, y en ese momento se puede decir que ha desarrollado la competencia.

Considerando que: "La competencia diferencia entre saber lo que hay que hacer en una situación determinada y el ser capaz de enfrentarse a ello en una situación real", (García y Paniagua, 1999).

El nuevo plan de estudios de secundaria, se intenta a través de la formación en competencias que el estudiantado aplique los conocimientos que adquiere en la vida cotidiana. Así mismo propone que se fomente la formación de actitudes y valores a fin de favorecer el desarrollo del alumnado para la convivencia pacífica con apego a la legalidad, el cuidado y respeto por el ambiente. También pretende que la educación que reciben sus alumnos y alumnas les permita dirigir su propio aprendizaje de manera permanente y con independencia a lo largo de su vida.

El planteamiento que ofrece el Acuerdo 384, concuerda con la conceptualización que Perrenoud (2004:11), emplea sobre competencia cuando señala que es "la capacidad de movilizar varios recursos cognitivos para hacer frente a un tipo de situaciones". El análisis de competencias remite constantemente a la teoría del pensamiento de la acción situada, que también se refleja en el mismo acuerdo cuando describe el apartado sobre recursos didácticos para dar soporte al desarrollo del plan de estudios que se relacionan con actividades vinculadas con problemas del entorno de las escuelas a través de la elaboración de proyectos. La cognición situada apoya el principio de realizar prácticas educativas auténticas que sean coherentes, significativas y propositivas, definidas como

"prácticas ordinarias de la cultura" (Brown, Collins y Duguid, 1989:34).

III. Formación en competencias y algunas implicaciones.

Las instituciones educativas cuando se preocupan por formar en competencias tienden a priorizar los recursos: capacidades y conocimientos. De alguna manera se preocupan por lo que serían parte de los ingredientes de las competencias, pero sus esfuerzos se minimizan al tratar de poner en movimiento estos recursos en situaciones complejas. Prueba de esto se encuentra a diario en la educación secundaria cuando se hace notorio el desfase entre los contenidos teóricos con los prácticos en un laboratorio.

A pesar de los esfuerzos que se ha realizado antes de la Reforma y se está llevando a cabo ahora, son insuficientes los logros alcanzados al tratar de conectar los conocimientos que imparte la escuela con situaciones que se dan en la vida cotidiana y los intereses de los adolescentes apenas alcanzó el $48.2 \%$ en un estudio realizado en los estados de

Hidalgo y Tlaxcala en el 2006 (Canales, 2006).

La noción de aprender se ha centrado más en dar respuesta a las exigencias de los contenidos programáticos que relacionarlos con la experiencia que viven sus alumnos y alumnas día a día.

El plan de estudios que se desarrolla en este momento en el último tramo de la educación básica propone un currículo único y nacional, que considera las distintas realidades de los alumnos. El problema es que, para implementarlo se requiere de estrategias flexibles de enseñanza y el uso de un repertorio 
muy amplio de recursos didácticos que están relacionados con el aprendizaje situado.

Para que una competencia se desarrolle es necesario contar con las capacidades y conocimientos para su transferencia y movilización, con el fin de aplicarla en distintas situaciones de su vida fuera del ámbito escolar.

Como se señaló anteriormente, en las escuelas se ha descuidado este aspecto por múltiples y complejas situaciones y se ha contentado el sistema con que los alumnos y alumnas acumulen conocimientos y pasen exámenes. Hay una cultura exacerbada por "pasar" más que por

"aprender" y esta situación impide que se movilicen los acervos aprendidos en situaciones de la vida, en el trabajo, la familia o las relaciones interpersonales (Canales, 2006).

Esta situación es especialmente difícil para los alumnos que se incorporan a edad temprana a la vida laboral, Perrenoud (2000), comenta que, cuando se formulan de manera prioritaria objetivos de formación en términos de competencias, se lucha abiertamente contra la tentación de la escuela: prepararse a sí mismo, marginar la referencia a situaciones de vida y no tomarse el tiempo de ayudar a la movilización de los acervos en situaciones complejas. Esto se puede ver con claridad en los resultados que se han obtenido en los últimos años en las pruebas de Pisa, cuyos indicadores se han estructurado a partir de problemas que se presentan en la vida cotidiana. Otro factor a considerar en este sentido, es el número de estudiantes que por diferentes razones no concluyen la secundaria, en el ciclo escolar 2006-2007. La eficiencia terminal fue de 78.8\% y de esta población que concluye el nivel de absorción a la educación media superior fue de $94.8 \%$. Esto nos plantea un panorama donde una proporción muy grande de alumnos que inician estudios en el último tramo de la educación básica y en la educación media superior no terminan y con frecuencia es porque tienen la necesidad de incorporarse a la vida laboral sin las competencias ni conocimientos suficientes.

III.1. Revisión de los propósitos de formación del currículo.

La descripción de las competencias en la planeación curricular tendría que partir de analizar las situaciones y de ahí derivar los conocimientos.

En el mapa curricular de la secundaria se contempla que, de primer a tercer año el $28.5 \%$ de las asignaturas corresponde a las materias instrumentales:

Español y Matemáticas, mientras que, en primero el $40 \%$ es de las materias sustantivas y el resto para Educación Física, Tecnología,

Arte, Asignatura Estatal y Orientación y Tutoría. En segundo y tercer año aumenta el porcentaje en las materias sustantivas a $40.5 \%$ al incorporar

Educación Cívica y Ética y disminuye en el resto al desaparecer la asignatura estatal.

Cuando se diseña un currículum por competencias se sugiere que se diseñe sobre núcleos problemáticos en torno a los cuales se integran varias disciplinas, de tal forma que se cuente con un currículo integrado.

En el caso del nuevo mapa curricular de la secundaria, hay una continuidad con la distribución de las asignaturas en la primaria, es así que en 
Ciencias I en el primer año se abordan contenidos de Biología, de Física en segundo y de Química en tercero, pero los conocimientos se siguen parcelando además por la forma en que son impartidos por diferentes docentes.

Diseñar un currículum por competencias requiere organizarlo a partir de procesos, y no sobre contenidos.

El plan de estudios de la secundaria, intenta resolver este problema con las metodologías que propone para su desarrollo y las formas de organización entre el profesorado. Es así que se espera que la propuesta promueva la convivencia y el aprendizaje en ambientes colaborativos y desafiantes y de esta manera se mejoren las relaciones entre maestros, alumnos y otros miembros de la comunidad. Para llevarla a su operatividad se considera que existen avances que se han gestado a partir de la Reforma de 1993. Entre ellas, se han abierto numerosas oportunidades para realizar proyectos didácticos compartidos entre maestros de diferentes asignaturas. Sobre todo en aquellos planteles donde la población no es numerosa y los docentes cubren la mayor parte de su tiempo en una sola escuela.

Se parte del hecho que el tipo de trabajo sugerido en la propuesta permita relacionar las actividades que desarrollan distintos maestros ya que sus contenidos están relacionados y parten de los intereses que en este momento de su vida tienen los adolescentes.

La concepción que se tenía de trabajo colegiado con esta propuesta se transforma y se hace necesario para compartir experiencias centradas en procesos de enseñanza y aprendizaje, considerando que el eje central de este proyecto es favorecer en los estudiantes la integración de saberes y experiencias desarrolladas en las diferentes asignaturas.

El diseño curricular de la secundaria plantea en el apartado de competencias para la vida la posibilidad de contribuir al mejoramiento de vida y a la convivencia en una sociedad que cada día se vuelve más compleja y por lo tanto, demanda la movilización de saberes culturales y la capacidad de aprender permanentemente para hacer frente a la creciente producción de conocimiento para que éste sea aprovechado en la vida cotidiana.

Reitera, como se planteó anteriormente, que el desarrollo de competencias implica un saber hacer (habilidades) con un saber (conocimiento), así como la valoración de las consecuencias del impacto de ese hacer (valores y actitudes).

Las competencias que propone, contribuirán al logro del perfil de egreso y son señaladas como una constante en todas las asignaturas:

a) Competencias para el aprendizaje permanente. Implican la posibilidad de aprender, asumir y dirigir el propio aprendizaje a lo largo de su vida, de integrarse a la cultura escrita y matemática, así como de movilizar los diversos saberes culturales, científicos y tecnológicos para comprender la realidad.

b) Competencias para el manejo de la información. Se relacionan con: la búsqueda, evaluación y sistematización de información; el pensar, reflexionar, argumentar y expresar juicios críticos; analizar, sintetizar y utilizar información; el conocimiento y manejo de distintas lógicas de 
construcción del conocimiento en diversas disciplinas y en los distintos ámbitos culturales.

c) Competencias para el manejo de situaciones. Son aquéllas vinculadas con la posibilidad de organizar y diseñar proyectos de vida, considerando diversos aspectos como los sociales, culturales, ambientales, económicos, académicos y afectivos, y de tener iniciativa para llevarlos a cabo; administrar el tiempo; propiciar cambios y afrontar los que se presenten; tomar decisiones y asumir sus consecuencias; enfrentar el riesgo y la incertidumbre; plantear y llevar a buen término procedimientos o alternativas para la resolución de problemas, y manejar el fracaso y la desilusión.

d) Competencias para la convivencia. Implican relacionarse armónicamente con otros y con la naturaleza; comunicarse con eficacia; trabajar en equipo; tomar acuerdos y negociar con otros; crecer con los demás; manejar armónicamente las relaciones personales y emocionales; desarrollar la identidad personal; reconocer y valorar los elementos de la diversidad étnica, cultural y lingüística que caracterizan a nuestro país.

e) Competencias para la vida en sociedad. Se refieren a la capacidad para decidir y actuar con juicio crítico frente a los valores y las normas sociales y culturales; proceder en favor de la democracia, la paz, el respeto a la legalidad y a los derechos humanos; participar considerando las formas de trabajo en la sociedad, los gobiernos y las empresas, individuales 0 colectivas; participar tomando en cuenta las implicaciones sociales del uso de la tecnología; actuar con respeto ante la diversidad sociocultural; combatir la discriminación y el racismo, y manifestar una conciencia de pertenencia a su cultura, a su país y al mundo (Acuerdo 384).

III.2.Implicaciones didácticas

Un buen ejemplo de los apoyos didácticos para alcanzar la apropiación de las competencias son las metodologías activas y aquí es importante advertir el riesgo que se corre de que los estudiantes se dispersen en diferentes actividades y entonces, se pierda la coherencia y la unidad de un horizonte conceptual.

Para desarrollar competencias es fundamental trabajar sobre todo por problemas y proyectos, como lo propone el plan de estudios de la secundaria.

Esto implica hacer propuestas de tareas complejas, enfrentar retos que inciten a los alumnos a movilizar sus acervos de conocimientos y habilidades y de alguna manera a completar lo que les falta. Llevar a cabo esta forma de operar el programa implica conocer, manejar y desarrollar en cada secundaria una pedagogía activa, cooperativa, abierta sobre la comunidad donde se inserta su escuela.

Es prioritario que los docentes tengan un dominio metodológico para trabajar los conceptos estructurales en función de los dominios cognitivos donde las estrategias docentes apunten hacia la interconexión de los temas.

III.3. Implicaciones en la evaluación

La evaluación ha sido un punto difícil de entender para los docentes de secundaria, resulta complicado hacer la ruptura entre calificar y evaluar. Con el Acuerdo 200 establecido desde 1994, se establece la normatividad para 
evaluar, pero no se especifica en realidad las implicaciones derivadas del concepto de evaluación formativa o sumativa ni se explica lo que se entiende por evaluación de procesos, simplemente indica: "evaluar el aprendizaje de los educandos entendiendo éste como la adquisición de conocimientos y el desarrollo de habilidades, así como la formación de actitudes, hábitos y valores señalados en los programas vigentes".

Este es un punto que se tendrá que atender en los siguientes ciclos escolares, considerando que, la evaluación por competencias esencialmente propone el cambio de una evaluación por logros a una evaluación por procesos. Por lo tanto, no se evalúa un resultado ni la suma de los aspectos considerados en una asignatura, se considera el proceso en el que se involucra el contexto, la motivación, los sistemas simbólicos y el desarrollo cognitivo. Ello implica hacer un seguimiento al proceso de aprendizaje desde la motivación hasta la ejecución de la acción y su consecuente resultado.

Se hace necesario evaluar con seriedad las competencias, considerando que es un proceso que se refiere a problemas complejos. La evaluación debe contribuir a que los estudiantes las desarrollen más. Exige la utilización funcional de conocimientos disciplinarios. La tarea a realizar y sus exigencias se necesitan conocer antes de la situación de la evaluación.

Esta modalidad demanda de la colaboración entre pares y considera estrategias cognoscitivas y metacognitivas utilizadas por los estudiantes (Perrenoud, 2000).

En el contexto de nuestras secundarias públicas, por las condiciones de trabajo de los docentes (tipo de plazas, exceso de grupos en diferentes planteles) se dificulta el trabajo colegiado que incluso propone la Reforma como un mecanismo para implementarla en cada escuela.

III.4. Metodologías de trabajo por competencias

Se contemplan básicamente tres metodologías para realizar el trabajo por competencias: proyectos, resolución de problemas y enseñanza para la comprensión.

- Trabajo por proyectos

Esta estrategia de enseñanza constituye un modelo de instrucción en el que los estudiantes planean, implementan y evalúan proyectos que tienen aplicación en el mundo real más allá del aula de clase. Se recomiendan actividades de enseñanza interdisciplinarias de largo plazo y centradas en el estudiante, en lugar de lecciones cortas y aisladas (Challenge

2000; Multimedia Project, 1999).

Dentro de las orientaciones didácticas que señala el Plan de Estudios de secundaria destaca que, en el desarrollo de proyectos, los alumnos se plantean cuestiones de diversa índole a nivel personal o social que responden a sus preguntas, necesidades y su propia acción social, fortaleciendo sus habilidades y actitudes. Entre éstas destacan el manejo de diversas fuentes de información, la realización y el cumplimiento de planes, el trabajo colaborativo, la capacidad de diálogo, la libertad, la tolerancia y el juicio crítico, la cooperación y el respeto que se ven reflejados en una mejor convivencia, la toma de decisiones, el diseño de objetos originales, la participación en grupos autónomos de trabajo, la comunicación efectiva y el 
uso de diferentes medios y lenguajes son otros aspectos que se ven fortalecidos (Acuerdo 384,14).

- Resolución de problemas

Esta metodología permite llevar a cabo una activación, promoción y valoración de los procesos cognitivos cuando los problemas y tareas se diseñan creativamente.

- Enseñanza para la comprensión

La enseñanza para la comprensión busca que los educandos se desarrollen como pensadores críticos, como personas que plantean y resuelven problemas y que sean capaces de enfrentar la complejidad del mundo en el que viven.

Lo anterior implica ir más allá de la rutina y vivir productivamente en este mundo que cambia rápidamente y se apoya con toda claridad en la convicción de que las escuelas deben comprometer a los alumnos de manera más decidida.

La enseñanza para la comprensión demanda de la organización de las imágenes y las representaciones mentales del alumnado en diferentes niveles para lograr la comprensión de los estudiantes y lograr que adquieran conciencia de su propio proceso. Trabajar con los diferentes niveles de comprensión, genera en el estudiante la capacidad propositiva y transformativa.

Estas metodologías demandan que el profesor debe dejar de pensar que dar cursos es el corazón del oficio. Enseñar hoy, debería consistir en concebir, establecer y controlar situaciones de aprendizaje, siguiendo los principios de las pedagogías activas y constructivistas (Perrenoud, 2000: 14).

\section{CONCLUSIONES}

La escuela secundaria afronta nuevos y significativos desafíos. Los cambios están ocurriendo en el interior de las aulas de los profesores y fuera de ellas por las transformaciones que se dan en el entorno. Se están produciendo cambios importantes en el paso de una cultura escrita a otra visual, dentro de un mundo de mayor complejidad tecnológica, se promueven cada vez más programas de atención y apoyo al estudiantado para alcanzar su bienestar social y emocional y mejorar la calidad educativa.

Es así que, formar en competencias desde la secundaria, es un acierto, considerando que en México un grupo numeroso de nuestra población difícilmente concluye la secundaria y al llegar al bachillerato deserta casi la mitad de la población que ingresa.

El problema de esta modalidad se encuentra en la dificultad que representa para los docentes el desarrollarla en su salón de clase. Por un lado implica una ruptura seria para aquellos que no poseen una visión constructivista e interaccionista del aprendizaje y por otra vencer la resistencia al cambio.

Trabajar de manera cooperativa y colegiada con los docentes que imparten clases a los grupos en los que ellos participan resulta una tarea que requiere de tiempo y disposición para el trabajo. La realidad laboral del docente de secundaria es, que en general, trabaja aislado porque su horario de trabajo se distribuye con frecuencia en diferentes planteles y atienden hasta 300 ó 400 alumnos a la semana en diferentes asignaturas. 
Los docentes de secundaria tendrían que hacer una autoevaluación sobre sus estrategias didácticas dentro del salón de clase, así como definir y valorar sus propias competencias como docentes y en su entorno. Esto demanda que den cuenta de lo que saben, conocen y manejan para buscar las alternativas de formación que son propias de cada caso.

Hace falta encontrar mecanismos auténticos para mejorar el sentido del trabajo escolar y modificar el dar cuenta del conocimiento de los alumnos que presentan dificultades para apoyarlos y no para descalificarlos.

Urge favorecer en todas las escuelas el abordaje de enfoques constructivistas así como formar ampliamente en tópicos relacionados con la evaluación formativa y la pedagogía diferenciada elementos centrales que favorecen la asimilación activa de los conocimientos.

El papel central de la Reforma de secundaria es la reflexión sobre las características y el papel de los jóvenes en la escuela secundaria y en la sociedad, por lo que resulta indispensable que los docentes y directivos tengan claridad sobre quiénes son los y las jóvenes que hoy estudian la secundaria y porqué la necesidad de formarlos con un currículo diseñado por competencias. Un docente y un directivo al que se le dificulta reflexionar, difícilmente lo va a enseñar en clase.

De lo anterior se desprende que el principal recurso del profesor, tendría que ser su postura reflexiva permanente y sistemática, su capacidad de observación, de control, de innovación, su disposición para aprender de otros: de los mismos alumnos, de la experiencia y desde luego su capacidad para organizar el trabajo escolar, para colaborar con sus colegas, para concebir diversos dispositivos pedagógicos, para crear y administrar situaciones, identificar problemas, obstáculos y tener la posibilidad de reencuadrar sus tareas.

Que comprenda lo que implica formar en competencias para la vida, ya que de ello depende que ésta sea una reforma más, donde gane puntos la burocracia, la resistencia, y la simulación.

"Lo esencial es saber qué buscamos, qué procesamos y qué hacer con ello. Hace falta inteligencia y capacidad de aprendizaje porque siempre estaremos aprendiendo, siempre, pero sólo si nos han enseñado cómo aprender. O sea, a escuchar, a pensar, a tener curiosidad." (Dominical El

Periódico 27.02.00)

FUENTES DE CONSULTA

- ACUERDO número 384 por el que se establece el nuevo Plan y Programas de Estudio para Educación Secundaria. Consultado el 10 de febrero 2007.

- ALONSO, L. (2000), “¿Cuál es el nivel o dificultad de la enseñanza que se está exigiendo en la aplicación del nuevo sistema educativo?” Revista EDUCAR, 26, pp. 53-74.

- BELTRAN LLERA, Jesús A. (2003). "De la Pedagogía de la Memoria a la Pedagogía de la Imaginación". En FUNDACIÓN ENCUENTRO; BELTRAN. - BROWN, J., Collins, A. y Duguid, P. (1989). Situated cognition and the culture of learning. Educational Researcher, 18 (1), 32-42 
- LLERA, J.A. (2003). La novedad Pedagógica de Internet. Madrid: Educared.

- CHALLENGE 2000 MULTIMEDIA PROJECT. (1999) consultado en: http://-www.education-world.com/awards/past/r1199-02.shtml el día 8 de marzo 2008.

- CANALES E., Vázquez G, Molina A. (2006), “Diagnóstico y lineamientos para la conformación de un modelo de atención tutorial en secundaria, reporte del proyecto de investigación: Diagnóstico Situacional para el diseño de un Modelo Tutorial en Secundarias Técnicas y Generales de los Estados de Hidalgo y Tlaxcala", 2003- C01-5/A-1 SEP/SEBYN-CONACYT 2003.

- CANALES E, Molina, A (2006), Teaching conditions in Mexican High School and tutelar attention en: "Vzgoja-Pre, Ministry of Education, Science and Sport of Republic of Slovenia. 33-38.

- CANALES E, (2006) "Scholar culture in secondary schools in Mexico in the states of Hidalgo and Tlaxcala" en: Comparative Education and Teacher training. Ed Bureau of Educacion Services and Bulgarian Comparative Education Society. 261-266.

- CAÑAL, P (1999) Investigación escolar y estrategias de enseñanza por investigación.

- DELORS, J. (1996) La educación encierra un divino tesoro. Correo UNESCO. México

- DÍAZ BARRIGA F, Rojas G (2001) Estrategias docentes para un aprendizaje

significativo. Mc Graw Hill, $2^{\mathrm{a}}$. Edición. México.

- DÍAZ BARRIGA F. (2003). Cognición situada y estrategias para el aprendizaje

significativo. Revista Electrónica de Investigación Educativa, 5

(2). Consultado el día 4 de marzo de 2008 en: http://redie.ens.uabc. $\mathrm{mx} / \mathrm{vol} 5 \mathrm{no} 2 /$ contenido-arceo.html

- DOMINICAL EL PERIÓDICO de Barcelona: 27.02.00.

- FIGUEROA M. (2006) La Reforma de la Educación Secundaria ¿Engaño o buenas Intenciones en: Observatorio Ciudadano consultado en:http:// observadormafq.blogspot.com/2006/09/la-reforma-de-la-educacinsecundaria.

html

- FENSTERMACHER, G. y Soltis, J. (1999), Enfoques de la Enseñanza. Buenos

Aires: Amorrortu.

- HARGREAVES, A.; Earl, L. y Ryan, J. (1998): Una educación para el cambio.

Reinventar la educación de los adolescentes. Barcelona: Octaedro. - MARTíNEZ N (2004) Modelos de enseñanza, Universidad de Murcia consultado en: http://209.85.173.104/search?q=cache:RsXN_pgvw_AJ: dewey.uab.es/pmarques/dioe/modelosnicolas.doc+Modelos+de+ense \% C3\%B1 anza $+\mathrm{P} \% \mathrm{C} 3 \% A 9 \mathrm{rez}+\mathrm{G} \% \mathrm{C} 3 \% \mathrm{~B} 3 \mathrm{mez} \& \mathrm{hl}=\mathrm{es} \& \mathrm{ct}=\mathrm{clnk \& cd}=4 \& \mathrm{gl}=\mathrm{mx}$, 
el 28 de febrero de 2008.

- PANIAGUA, G. (1999). Las familias y los niños con necesidades educativas especiales. En A. Marchesi , C. Coll y J. Palacios. Desarrollo psicológico

y educación. 3. Trastornos del desarrollo y necesidades educativas especiales. (pp. 469-493), Madrid: Alianza.

- PERRENOUD PH (2000), El arte de construir competencias. Brasil: Noval Escola.

(2004 ), Diez nuevas competencias para enseñar. España:Grao

(2007) Desarrollar la práctica reflexiva en el oficio de enseñar. España:

Grao

- PLAN NACIONAL DE DESARROLLO 2007-2012 Problemas y propuestas en materia educativa. Consultado en: http://209.85.173.104/

search?q=cache:bx3_FzsqohgJ:www.derechosinfancia.org.mx/Documentos/

pnd_educacion.pdf+eficiencia+terminal+de+secundaria+nac

ional + ciclo $+2005+2006 \& h l=e s \& c t=c l n k \& c d=4 \& g l=m x, 10$ de marzo

2008.

- TRABAJO POR PROYECTOS, consultado.

http://www.eduteka.org/AprendizajePorProyectos.

php, el 6 de marzo 2008.

-TAPIA, Jesús Alonso (2000) Motivación y Aprendizaje en el aula, Cómo enseñar a pensar, Editorial Santillana, Madrid. Aula XXI, Num 50.

-TÉBAR Belmonte, L (2003), El perfil del profesor mediador. Madrid:

Santillana. 